\title{
Wetting the whistle: neurotropic factor improves salivary function
}

\author{
Adam Swick ${ }^{1}$ and Randall J. Kimple $e^{1,2}$ \\ 'Department of Human Oncology and '2arbone Comprehensive Cancer Center, School of Medicine and Public Health, University of Wisconsin, Madison, Wisconsin, USA.
}

\begin{abstract}
Xerostomia, or dry mouth, is a common side effect of head and neck radiotherapy, Sjögren syndrome, diabetes, old age, and numerous medications. In this issue of the $J C I$, Xiao and colleagues identified glial cell line-derived neurotrophic factor (CDNF) as a potential stimulus for salivary stem cell growth. Due to its ability to promote neuronal growth, differentiation, and survival, GDNF is currently being used in clinical trials as a treatment for Parkinson disease; therefore, the findings of Xiao and colleagues may initiate a potential treatment for the millions of patients who suffer from xerostomia each year.
\end{abstract}

\section{Multiple causes of a dry mouth}

Dry mouth, or xerostomia, is the result of inadequate salivary function and subsequent inability to keep the mouth moist and properly lubricated. Chronic xerostomia can dramatically affect a patient's quality of life, as it leads to difficulty chewing, eating, swallowing, and speaking. Furthermore, the reduction of important salivary enzymes, which aid in initial digestion of food, can result in dysgeusia (i.e., altered taste) in xerostomia sufferers. Oral health problems, including oral candidiasis, periodontal disease, and tooth decay, can also arise as a consequence of long-term xerostomia. While most individuals experience occasional xerostomia as a result of stress or anxiety, this shortterm symptom only affects patients for a finite time period. Unfortunately, a subset of people suffer from chronic xerostomia as the result of medications, such as antihypertensives, antidepressants, antihistamines, and diuretics, among others, as well as diabetes, Parkinson disease, Sjögren syndrome, and as a side effect of head and neck radiotherapy (1).

Each year in the US, 40,000 patients are diagnosed with head and neck cancer, and many of these patients are treated with radiotherapy at some point during their care. While older, 3D conformal radiation results in long-term xerostomia in up to $80 \%$ of patients, use of intensitymodulated radiotherapy (IMRT) can decrease the xerostomia rate to $20 \%$ to $30 \%$ (2). Patients left with radiationinduced xerostomia can develop compromised dentition, impaired swallowing ability, and difficulty speaking. For patients that develop xerostomia, the associated symptoms can have a major negative impact on the overall quality of life and hinder the recovery process.

Importantly, xerostomia potentially affects a much larger cohort of patients who have one of several chronic illnesses. Over one million patients per year in the United States suffer from Sjögren syndrome and other associated autoimmune disorders; many of these experience xerostomia symptoms. (1). There is also evidence that patients with poorly controlled diabetes frequently suffer from xerostomia. The incidence of diabetes has reached near epidemic proportions in the United States with over 25 million diagnosed cases, and more than $10 \%$ of diabetics may suffer from extreme hyposalivation (defined by a resting salivary flow rate $<0.01 \mathrm{ml} / \mathrm{min}$ )

Related Article: p. 3364

Conflict of interest: The authors have declared that no conflict of interest exists

Reference information: / Clin Invest. 2014;124(8):3282-3284. doi:10.1172/JCI77194.

Many more diabetic patients complain of dry mouth or difficulty swallowing, which are typical of milder xerostomia (3).

Finally, a plethora of commonly prescribed mediations can result in xerostomia. While the exact number of patients who suffer from medication-induced xerostomia is not known, the widespread use of medications that promote xerostomia suggests that a significant number of patients are affected by this condition.

\section{Current treatment options}

Treatment options for radiation-induced xerostomia focus on avoiding or ameliorating damage to the major salivary glands (Table 1). IMRT uses multiple beams and modulates intensity to limit the dose of radiation to the parotid or submandibular glands. Surgical transposition of the submandibular glands has also been used to move a gland out of the radiation field during the course of radiotherapy and return the gland once radiation therapy is complete $(4,5)$. The pharmacologic agent amifostine is used to mitigate the effects of radiation and must be delivered daily via i.v. injection throughout the course of radiotherapy. Unfortunately, amifostine only provides modest benefits, but causes substantial side effects $(6,7)$.

Treatments for symptomatic xerostomia are generally supportive in nature. For example, patients are encouraged to increase water consumption by keeping a water bottle present at all times and to specially prepare food to aid swallowing (8). Several pharmacologic treatments are available to increase salivary production in patients with normal salivary acinar structure. Pilocarpine is a nonselective muscarinic receptor agonist that stimulates the secretion of saliva and sweat (9), and salivary substitutes are marketed by several companies, such as Biotene, which produces a sodium monofluorophosphatecontaining line of oral care products. While these products successfully relieve xerostomia symptoms in some patients, 


\section{Table 1. Current treatments for xerostomia}

$\begin{array}{lc}\begin{array}{l}\text { Treatment } \\ \text { Salivary substitutes } \\ \text { Salivary stimulants }\end{array} & \text { Water, artificial saliva, milk, oil } \\ \text { Salivary transplantation } & \begin{array}{c}\text { Organic acids (ascorbic acid, malic acid), parasympathomimetic drugs } \\ \text { (pilocarpine, cholinesterase inhibitors), chewing gum, sugar-free mints }\end{array} \\ \begin{array}{l}\text { Salivary-sparing radiation } \\ \text { Stem cell transplantation } \\ \text { Viral therapy }\end{array} & \text { Relocation of salivary gland out of radiation field } \\ \text { IMRT }\end{array}$

controlled clinical studies reveal mixed responses $(10,11)$. The primary limitation of these approaches is that they fail to address the underlying cause of xerostomia in many patients, inadequate or malfunctioning salivary cells.

\section{Restoring normal salivary function}

Unsurprisingly, the development of therapies to help restore normal salivary function in patients suffering from xerostomia is of great clinical interest. Recently, Baum and colleagues reported the use of adenoviral-mediated delivery of genes encoding the water channel aquaporin-1 into the salivary gland, which provided objective improvement in salivary production in about half of the subjects (12). This type of adenoviral approach has the potential to provide a more permanent treatment of this symptom; however, the expected duration of improvement following transgene delivery and other challenges associated with viral gene therapy remains unknown. Intriguingly, transplantation of salivary stem cells within bioengineered organs has also shown promise in rat and mouse models of xerostomia (13-15). However, this powerful approach remains years away from clinical implementation due to limited sources of human stem cells.

In this issue, Xiao and colleagues built on earlier work demonstrating that transplantation of salivary stem cells into irradiated glands can improve hyposalivation $(16,17)$. Xiao et al. successfully identified and isolated a population of $\operatorname{Lin}^{-} \mathrm{CD} 24^{+}$ c-Kit ${ }^{+} S c a 1^{+}$stem cells in the submandibular glands of adult mice, which improved salivary gland condition and increased salivation when transplanted into irradiated animals (18). While attempting to uncover the critical pathways involved in the activity of this salivary stem cell population, Xiao and colleagues made an exciting finding that has the potential to affect current xerostomia treatment. Gene-expression analysis revealed that a subpopulation of cells highly expressed glial cell line-derived neurotrophic factor (GDNF). Importantly, mice given GDNF exhibited enhanced function and integrity of irradiated salivary glands without any apparent effects on head and neck tumor growth. While stem cell-based therapies likely will require the implantation of isolated stem cells to repopulate the affected gland, the results of Xiao et al. suggest a pharmacologic approach for improving salivary gland function. Although the motivation for their study was to improve salivary gland function following radiation, the findings of Xiao and colleagues have potential implications for the millions of Americans who suffer daily from chronic xerostomia that did not develop as the result of head and neck radiotherapy.

\section{Conclusions}

While the results of Xiao and colleagues are exciting, this study represents only a first step along the way to improved treatments for xerostomia patients. It remains to be determined whether GDNF treatment can protect salivary function for a multiweek course of fractionated radiation and how many GDNF treatments are required for long-term improvements in salivary function. Furthermore, the optimal timing of GDNF treatment (before or after the development of xerostomia) in non-radiation-induced xerostomia remains uncertain. Importantly, patients undergoing radiation therapy may represent a small minority of patients suffering from xerostomia who could potentially benefit from GDNF administration. Therapies that are efficacious, cost effective, and easily administered have the potential to improve quality of life for millions of patients. Perhaps the results from Xiao and colleagues are the first trickle of a cascading waterfall of progress for patients suffering from xerostomia.

\section{Acknowledgments}

This work was supported by CA160639.

Address correspondence to: Randall J. Kimple, Department of Human Oncology, Carbone Comprehensive Cancer Center, School of Medicine and Public Health, University of Wisconsin, Madison, Wisconsin 53705, USA. Phone: 608.265.9156; E-mail: rkimple@humonc.wisc.edu.

1. von Bultzingslowen I, et al. Salivary dysfunction associated with systemic diseases: systematic review and clinical management recommendations. Oral Surg Oral Med Oral Pathol Oral Radiol Endod. 2007;103(suppl):S57.e1-S57.e15.

2. Marta GN, et al. Intensity-modulated radiation therapy for head and neck cancer: systematic review and meta-analysis. Radiother Oncol. 2014;110(1):9-15.

3. Moore PA, Guggenheimer J, Etzel KR, Weyant RJ, Orchard T. Type 1 diabetes mellitus, xerostomia, and salivary flow rates. Oral Surg Oral Med Oral Pathol Oral Radiol Endod. 2001;92(3):281-291.

4. Kolahi J, Mansourian M. Autotransplantation of cryopreserved minor salivary glands: a new approach for management of radiation-induced xerostomia. Med Hypotheses. 2010;74(1):29-30.

5. Jha N, et al. Prevention of radiation induced xerostomia by surgical transfer of submandibular salivary gland into the submental space. Radiother Oncol. 2003;66(3):283-289.

6. Haddad R, et al. Randomized phase 2 study of concomitant chemoradiotherapy using weekly carboplatin/paclitaxel with or without daily subcutaneous amifostine in patients with locally advanced head and neck cancer. Cancer. 2009;115(19):4514-4523.

7. Jellema AP, et al. Radiotherapy alone, versus radiotherapy with amifostine 3 times weekly, versus radiotherapy with amifostine 5 times weekly: a prospective randomized study in squamous cell head and neck cancer. Cancer. 2006;107(3):544-553.

8. Visvanathan V, Nix P. Managing the patient presenting with xerostomia: a review. Int JClin Pract. 2010;64(3):404-407.

9. Gornitsky M, et al. Double-blind randomized, placebo-controlled study of pilocarpine to salvage salivary gland function during radiotherapy of patients with head and neck cancer. Oral Surg Oral Med Oral Pathol Oral Radiol Endod. 2004;98(1):45-52. 
10. Aliko A, Alushi A, Tafaj A, Isufi R. Evaluation of the clinical efficacy of Biotène Oral Balance in patients with secondary Sjögren's syndrome: a pilot study. Rheumatol Int. 2012; 32(9):2877-2881.

11. Shahdad SA, Taylor C, Barclay SC, Steen IN, Preshaw PM. A double-blind, crossover study of Biotene Oralbalance and BioXtra systems as salivary substitutes in patients with post-radiotherapy xerostomia. Eur J Cancer Care (Engl). 2005;14(4):319-326.

12. Baum BJ, et al. Early responses to adenoviral- mediated transfer of the aquaporin-1 cDNA for radiation-induced salivary hypofunction. Proc Natl Acad Sci U S A. 2012;109(47):19403-19407.

13. Jeong J, et al. Human salivary gland stem cells ameliorate hyposalivation of radiation-damaged rat salivary glands. Exp Mol Med. 2013;45:e58.

14. Ogawa M, et al. Functional salivary gland regeneration by transplantation of a bioengineered organ germ. Nat Commun. 2013;4:2498.

15. Lim JY, et al. Systemic transplantation of human adipose tissue-derived mesenchymal stem cells for the regeneration of irradiation-induced sali- vary gland damage. PLoS One. 2013;8(8):e71167.

16. Feng J, van der Zwaag M, Stokman MA, van Os $\mathrm{R}$, Coppes RP. Isolation and characterization of human salivary gland cells for stem cell transplantation to reduce radiation-induced hyposalivation. Radiother Oncol. 2009;92(3):466-471.

17. Lombaert IM, et al. Rescue of salivary gland function after stem cell transplantation in irradiated glands. PLoS One. 2008;3(4):e2063.

18. Xiao N, et al. Neurotrophic factor GDNF promotes survival of salivary stem cells. JClin Invest. 2014;124(8):3364-3377. 